Glocal Business: Método Gerencial para la Glocalización de negocios http://doi.org/10.33996/revistaenfoques.v1i4.20 No. 4 | Volumen 1 | Octubre - Diciembre 2017 http://revistaenfoques.org ISSN: $2616-8219$

\section{GLOCAL BUSINESS: MÉTODO GERENCIAL PARA LA GLOCALIZACIÓN DE NEGOCIOS}

\author{
GLOCAL BUSINESS: METHOD MANAGEMENT FOR THE \\ GLOCALIZATION OF BUSINESS
}

Adriana Barrera Rodríguez y Marcos Fidel Barrera Morales

\begin{abstract}
Resumen
Los fenómenos de la globalización, la apertura internacional de mercados y de las organizaciones -públicas y privadas-, plantean la necesidad de formas administrativas y gerenciales que permitan asumir estas circunstancias como estrategia, a fin de garantizar sobrevivencia, desarrollo y apertura de oportunidades y negocios. Esta propuesta corresponde a un trabajo especial de grado, de abordaje caológico, exógeno (Hurtado, 2010) y de diseño y fuente documentales. Se aplicaron técnicas de análisis por relaciones, prefigurativas de escalamiento y de análisis composicional (Barrera, 2006). Además, se determinó un esquema de trabajo mediante el cual se estudiaron y argumentaron, in extenso, contenidos generales relacionados con los negocios y los procesos de internacionalización y se estudiaron aspectos esenciales científicos y estructurales. También, se estructuró la propuesta metodológica gerencial denominada Glocal business. Esta contiene orientaciones para la migración administrativa y gerencial y la creación de condiciones operativas que faciliten este tránsito organizacional, así como la sostenibilidad de las propuestas en el contexto de las exigencias de la denominada glocalización.
\end{abstract}

Palabras clave: Administración; estrategia empresarial; globalización; glocalización; internacionalidad

\begin{abstract}
The phenomena of globalization, the international opening of markets and the possibilities of organizations - companies, entities, public and private -, propose the need to structure administrative and managerial forms that allow to assume these circumstances, as a strategic resource, in order to Guarantee the survival, development and opening of business and opportunities. The present proposal corresponds to a special work of degree, of caological, exogenous approach and of design and documentary source. Pre-figurative scaling techniques, relationship analysis technique and compositional analysis technique were applied for its realization. In this special work was determined a general scheme of work in the first part of which were studied and argued extended on general contents related to business and internationalization processes and studied essential scientific and structural aspects. In the second part, a proposal was proposed that suggests a managerial methodology called Glocal business. The proposed methodology contains guidelines for administrative and managerial migration, and the organizational creation of operational conditions that facilitate this transit, as well as the sustainability of the proposals in the context of the demands of the so-called glocalization.
\end{abstract}

Key words: Administration; business strategy; globalization; glocalization, internationality

\section{Adriana Barrera Rodríguez}

adriana.barrerarodriguez@gmail.com Euroamerican Learning University, Curazao

Contador Público de la Universidad Católica Andrés Bello. Magister en Gerencia. Diplomado en Gerencia de proyectos. Asesor y consultor empresarial con desarrollada experiencia.

\section{Marcos Fidel Barrera Morales} marfibamo@outlook.com

Centro Internacional de Estudios Avanzados Sypal, Venezuela

Licenciado en Comunicación Social de la Universidad Católica Andrés Bello, Magister en Filosofía. Doctor en Filosofía. Director General de Ciea Cypal. 


\section{INTRODUCCIÓN}

La internacionalización, como fenómeno que conlleva a la expansión de la actividad comercial, gerencial y productiva, a la diversificación geográfica y a la sobrevivencia de las empresas, debe estar considerada a lo largo de la vida de las organizaciones. Las organizaciones se ven afectadas por el impacto de la internacionalización y por los aspectos que la caracterizan. De ahí que su estudio es importante, para orientar las acciones de la empresa, ya sea en el mercadeo de productos, bienes y servicios de manera competitiva, o para en cuanto a la orientación organizacional hacia contextos de mayor demanda. De igual manera, es fundamental considerar el impacto de la internacionalización en la obtención de productos y materias primas que permitan mantener y mejorar condiciones de producción y comercialización de la empresa. Las naciones se desarrollan en las relaciones mutuas, gracias a los acuerdos y a los nexos que se instituyen. Asimismo ocurre con las empresas, con las organizaciones y las experiencias productivas, entre sí, también están marcadas por el imperativo nacional y regional que favorece la actividad trasnacional.

Entre los asuntos que derivan de este fenómeno se desprende la necesidad de competir más audazmente, ya que, aunque la empresa no salga a escenarios mundiales o se niegue a la internacionalización, su mercado de competencia se ve afectado por las empresas que abarcan el mercado local. De igual manera están influenciadas por la presencia de empresas de otras latitudes en su propio contexto de acción. Por ello, se tienen dos opciones: o nadar en el mar de la internacionalización o dejarse arropar por el fenómeno y correr los riesgos que esto implica.
Desde un punto de vista optimista, la internacionalización ofrece la ventaja de abrir un abanico de oportunidades hacia mercados que por sus características pueden muy bien aceptar ideas y conceptos de negocios, donde seguramente las necesidades en una rama productiva no han sido cubiertas en su totalidad o no han sido aprovechadas ni explotadas. De igual manera contribuye a que empresas a punto de extinción, como resultado de las limitaciones del mercado donde se desenvuelven, encuentren nuevas opciones para operar desde otros ámbitos. También es una oportunidad para empresas que por su conveniencia en el intercambio de conocimientos y de productos para sus servicios y soluciones, requieren ubicaciones estratégicas para obtener productos primarios, adquirir y desarrollar nuevas tecnologías y proveerse de materia primas.

La importancia de los negocios en la línea de la internacionalización se traduce en la capacidad de extender en diversos territorios, servicios productos o soluciones, no sólo que satisfagan necesidades cubiertas en otras regiones sino que faciliten la vida de los seres humanos, generen nuevas opciones de empleo y combinen múltiples conocimientos y prácticas de trabajo entre regiones distintas y a la vez interconectadas entre sí.

Los negocios, en cualquiera de sus formas, son el motor que impulsa la capacidad productiva y económica de una nación, cuando logran ser materializados. Esta capacidad productiva y económica, a su vez, promueve la capacitación y profesionalización, el surgimiento de empresas, las posibilidades de empleo y el desarrollo social, de una nación. Los negocios son el encuentro entre capacidades, conocimientos y oportunidades. Cada negocio productivo es el resultado de 
engranar estas variables y materializar un sueño o un ideal, que además de satisfacer a quien lo promueve tanto profesional como económicamente, puede otorgar beneficios a muchas personas, por aquello que produce, sean cosas o servicios. Los negocios también contribuyen con la base de las economías de los municipios, estados, naciones, regiones, continentes y del mundo entero.

Los negocios producen la mayor parte de los bienes y servicios que la gente consume y emplea. Crean muchas innovaciones y proporcionan un amplio rango de oportunidades para abrir negocios nuevos, que a su vez sirven como sus proveedores. Un clima de negocio saludable también contribuye directamente a la calidad y al estándar de vida. Nuevas formas de tecnología de negocios de servicio y oportunidades internacionales prometen conservar la producción, el consumo y el crecimiento del empleo de manera indefinida. Las ganancias de los negocios fomentan los ingresos personales de millones de propietarios y accionistas, y los impuestos de estos negocios apoyan a los gobiernos en todos sus niveles. Muchos negocios apoyan a instituciones de beneficencia y le imprimen liderazgo a su comunidad (Griffin y Ebert., 2005, p. 4).

La empresa representa una forma seria de representar y formalizar una idea de negocio, con intenciones claras de figurar en la economía, de comprometerse con la actividad económica local, regional, nacional o mundial. La actividad empresarial es soporte económico y social. "El comportamiento empresarial es el motor fundamental de la autorenovación de la economía. Los empresarios reconocen las oportunidades de mercado y reasignan los recursos disponibles de manera más eficiente" (Sánchez, et al., 2014, p. 278).
En la perspectiva de los negocios existe un escenario mundial y planetario, que cada vez se amplía, y que además, con herramientas tecnológicas adecuadas, supera distancias, idiomas y limitantes, a fin de ayudar a generar iniciativas empresariales que obligan a pensar en la idea de asentar operaciones en lugares con distintas condiciones, o de buscar la ampliación de operaciones que compensen los efectos que vive cada empresa en su lugar de origen.

Se habla, entonces, en términos de la globalización y de la atención a lo local, como dos expresiones de una misma realidad. Lo local es lo cercano. La globalidad es lo regional, mundial, con soporte tecnológico.

La experiencia que tiene cada empresa en el contexto donde se desenvuelve, local, regional, nacional o internacional, es de importancia para esa decisión, pues, más que intentar replicar una experiencia, se trata de sacarle máximo provecho a los saberes, a fin de explotar las mejores condiciones internas y externas dentro de cualquier glocalidad.

La globalización es una realidad que no se escapa de las críticas de la humanidad, ya que se han podido cuantificar ventajas $y$ desventajas de distinta índole. A través de las diferencias sociales y políticas, además de las económicas, está presente el riesgo de las minorías privilegiadas, en contra de las mayorías desfavorecidas. De igual manera afecta la llamada identidad cultural, de regiones y países. Por esto, se justifica el surgimiento de leyes regulatorias y de políticas proteccionistas de las culturas, igual de las economías y de las sociedades, para poner normas de intercambio multicultural. Ciertamente, en algunos aspectos como el 
económico y el cultural ha sido la globalización un proceso agresivo, mayormente aprovechado por algunos, por lo que se espera todavía la oportunidad de las inmensas mayorías mundiales.

La decisión más conveniente, al frente de la globalización, consiste en nadar en favor de la corriente económica, social y tecnológica, con base en el análisis de las ventajas que conlleva el proceso globalizador así como también tener en cuenta las pautas legales, políticas y económicas. Esto, cuando se está sujeto a ser afectado negativamente - cuando se teme perder algún valor económico o cultural de valor propio para la nación.

Desde la perspectiva global, el desarrollo de cualquier iniciativa viene seguida del pensamiento relacionado con el crecimiento internacional. Este crecimiento se basa también en el reconocimiento de las oportunidades que cada contexto brinda y del efecto de la propuesta que cada entidad ofrece al entorno económico y social, en el escenario mundial donde desea incursionar. Este tipo de crecimiento, tal como lo requiere el mundo globalizado, es resultado de la práctica de llevar a cabo nuevos emprendimientos.

Por otra parte, a partir de las últimas décadas, los negocios cada día dependen, más que de la diferencia entre los productos que ofrecen, del contexto, y de la perspectiva servicial, que cumplen. Este factor servicio está vinculado con el estilo y la manera humanos y organizacionales. Por ello, está en correspondencia con las actitudes, con las aptitudes, con las capacidades, con las potencialidades $y$, en general, con la fuerza productiva, en toda su capacidad y formación.

Cuando se busca un bien, producto o servicio se pueden encontrar múltiples oferentes, cada uno de los cuales plantea productos similares, en condiciones diferentes. Por ello, se dice que se está en presencia de un mercado competitivo para el que ofrece, donde el que oferta el producto o servicio con mayores ventajas competitivas al cliente es quien reporta el cierre de un negocio, al proporcionar, en consecuencia, mayores beneficios. El emprendedor que se organice, se prepare, invierta y oriente su misión y visión de la forma más clara posible, reporta mayor productividad.

Si bien los agentes impulsadores de los procesos de internacionalización están presentes en la dinámica local y mundial, los emprendedores interesados no siempre saben evaluar si las condiciones son aptas, así como y por dónde empezar. Por ello, hacen falta servicios de orientación para inversionistas, para emprendedores y para desarrolladores de proyectos empresariales, interesados en la proyección internacional. Por esa razón, este trabajo tuvo como objetivo precisar una propuesta estructural y organizacional de internacionalización de negocios en el contexto de la glocalización, aplicable a diferentes tipos de organización.

Glocal business surge como una propuesta ante la gran necesidad de contar con orientaciones gerenciales prácticas y aplicables por emprendedores de cualquier naturaleza, interesados en proyectos de internacionalización de negocios. Obedece, a la tarea propuesta de organización de orientaciones formales, en torno a los procesos gerenciales, también de liderazgo y dirección de procesos.

Se entiende por empresa una entidad institucional en su calidad de productora de bienes y servicios. Una empresa es un agente económico con autonomía para adoptar decisiones financieras y de inversión y con autoridad y responsabilidad para asignar 
recursos a la producción de bienes y servicios y que puede realizar una o varias actividades productivas (Naciones Unidas, 2009, p. 34).

La labor central de toda empresa es la negociación, ya sea de productos o de servicios. La negociación es un proceso comunicacional, estratégico, que puede ser aplicado en diversos contextos, incluso en los conflictos políticos, sociales y personales. Aunque su resultado no siempre apunte a un beneficio económico, necesariamente es una herramienta muy empleada en su consolidación. Para muchos la negociación es un arte y conlleva al manejo de destrezas comunicacionales. Se está en presencia de una negociación cuando coinciden intereses, entre las partes involucradas $y$, necesariamente, se fijan principios de acuerdo, en aspectos no concordantes.

El sustantivo castellano negocio procede del latín negotium. Esta palabra, a su vez, está formada por la negación nec y el sustantivo otium 'ocio': nec + otium > negotium (literalmente, 'lo que no es ocio'). Es una forma curiosa de conceptualizar esta realidad extralingüística. Para los romanos los negocios u ocupaciones son lo que hacemos cuando no estamos disfrutando de nuestro tiempo libre (Bustos, 2014, párrafo 1).

El humano, en su afán de innovar y ser cada día más competitivo, da origen a diversas formas de negocio en los cuales, finalmente, ofrece cada vez más valor agregado a sus productos y servicios. Existe las posibilidades de negociar cualquier producto, servicio e insumo sin importar el lugar donde cada quien se encuentre productor e interesado-. De igual manera las distintas monedas en las que se puede pagar, luego se trata de resolver el tiempo que tarda en llegar y la forma de trasladarse lo ofertado. En el caso de los servicios, se aprecia cómo es posible prestar cualquier servicio desde cualquier lugar del mundo, con el apoyo del internet y las diferentes herramientas tecnológicas. Esta diversidad en la forma de proponerse un negocio es lo que se denomina modelo de negocios, en el cual se "describe las bases sobre las que una empresa crea, proporciona y capta valor" (Osterwalder y Pigneur, 2011, p. 14).

La Negociología es una ciencia social que, a través de un conjunto de conocimientos estructurados, propone de una manera lógica, alternativas para que tanto el ser humano y su familia, como el empresario y los recursos humanos de su compañía (considerados como núcleo familiar a nivel corporativo), puedan progresar y prosperar (Brizuela, 2016, párrafo 3).

Las empresas representan la estructura mínima formal productiva en cada contexto económico, y el conjunto de ellas son las que conforman el motor productivo de una economía. Las empresas son las principales generadoras de empleo en la sociedad, como también fuente de ingresos para los estados nacionales y fundamento del progreso general de un país. Constituyen "aquella unidad que dispone de unos determinados factores de producción, los cuales se combinan por decisión del hombre, para conseguir unos productos y servicios que se venden." (García, 1974, c.p., 29). A su vez, es: un conjunto de medios humanos $y$ materiales que se disponen para conseguir una finalidad según un esquema determinado de relaciones y dependencias dentro de los diferentes elementos que la componen (Zerilli, 1992 c. p., 30).

Las empresas pueden tener alcance local, regional, nacional, internacional y pueden adecuar las estructuras organizativas y de desempeño, de acuerdo al alcance de las 
operaciones. Un grupo importante de ellas tienen excepcional trascendencia, con poca estructura organizacional. A cualquier parte donde desean llegan, basados en herramientas tecnológicas y comunicacionales, propias del contexto globalizado.

Hoy por hoy, ante los cambios globales, surge un aspecto importante de valorar en las empresas, que también permite otro nivel de clasificación organizacional. Se habla, entonces, de las empresas innovadoras, como aquellas que se basan en el potencial de conocimientos, con estructura comunicacional y de operaciones netamente tecnológica, que responden a un alto nivel de innovación. Para Sakaya (1995) estas empresas tienen compenetración con las universidades, como fuente de investigación y desarrollo, lo cual va muy de la mano de las algunas tendencias de inscritas en la sociedad de conocimiento.

Estas características no son fáciles de cuantificar y valorar, pero son reflejadas en la versatilidad de sus actividades, en la velocidad de crecimiento, en la poca estructura física y en la capacidad de innovación. Este es el caso de empresas que manejan una actividad especializada, o aquellas que tienen patentes de industria, como también aquellas dedicadas a la microbiología, a la biogenética, a las telecomunicaciones, la tecnología. Estas satisfacen mercados de alta demanda, con eficiencia, y se les adjudica un buen tiempo de vida.

Los rasgos que caracterizan a la globalización, a través de la economía mundial, desde el siglo pasado, son descritos por Calva y Álvarez (2007), quienes comentan que en el siglo anterior las potencias hegemónicas eran distintas $y$ jugaban también papeles importantes. A su vez, la movilidad migratoria de trabajo de hace un siglo fue mayor, pues el siglo XXI caracteriza a la globalización por el surgimiento del estado bienestar, que busca estabilizar con políticas de estado los estragos de la reciente globalización.

A su vez, durante el último siglo se destacan tres etapas de la globalización: La primera desde finales del siglo XIX hasta la segunda guerra mundial, caracterizada por fuerte crecimiento del comercio $y$ denominada edad de oro del comercio y la inversión; la segunda, desde la década de los 50 hasta los 70, caracterizada por un menor crecimiento de la productividad; la tercera se caracteriza por: la aparición de la nuevas tecnologías, la internacionalización de los mercados financieros, el incremento del comercio interindustrial, la aparición de empresas globales, la desregulación de países miembros y la apertura a países no miembros, de igual manera que la existencia de nuevos tipos de organización flexible en la producción.

A lo largo de la historia la globalización ha tenido diversas interpretaciones en la medida de los avances de la sociedad y resultado de las adaptaciones a los procesos de cambio de la humanidad. La humanidad en cada uno de sus procesos de cambio ha tenido la intención de corregir las vicisitudes $y$ peligros de estas relaciones 0 interconexiones de la sociedad, en cada uno de sus aspectos: sociales, económicos, políticos y culturales. Estas relaciones han traído consecuencias positivas, sin dejar de reconocer los desequilibrios que también proporcionan.

$\mathrm{Si}$ las empresas nacionales logran desarrollar una importante innovación gracias al apoyo gubernamental mediante subsidios a la I+D tendrán posibilidades de bloquear la entrada a sus potenciales rivales extranjeros gracias a la reducción de costes 
(recuérdese el modelo del precio límite presentado anteriormente) o al desarrollo de productos totalmente nuevos derivados de la investigación (Steimberg, 2004, p. 67).

Uno de los retos de las empresas en el proceso de globalización es ser competitivas frente a empresas de otras regiones que pueden ofrecer diversas ventajas a los posibles clientes. Se entiende por competitividad a la capacidad de quienes ofrecen bienes, productos y servicios para destacarse en distintos ambientes, al resaltar las ventajas comparativas. Es considerada el motor que mueve los mercados, alimenta la oferta y la demanda y promueve la libre competencia. En los mercados competitivos prevalecen los entes que no se doblegan ni se opacan ante sus adversarios, que al contrario luchan por destacarse al tomar en cuenta las ventajas comparativas. Que además se apoyan en la innovación para mantenerse frescos y vivos ante la competencia de los mercados, tanto desde el punto de vista de la originalidad de sus productos como de sus precios.

La competitividad es una característica de las empresas que se promueve no sólo con los aportes tecnológicos y de innovación. Para generar competitividad también se requiere la inclusión de formas de gestión y organización, la inversión en investigación y desarrollo y vigilancia de la información. La competitividad debe ser desarrollada desde una perspectiva global, de tipo sostenible y de largo plazo, que considere el entorno macroeconómico y microeconómico, ya que la misma debe ajustarse para abordar los mercados internacionales y para afrontar el bombardeo de la globalización en su propio contexto local.

La dirección estratégica de las empresas es un factor crucial para entrar de manera competitiva al mercado global. Es oportuno mencionar a Porter (2016) como uno de los aportes teóricos que han sido incorporados a la dirección estratégica de empresas.

CEPAL y UNESCO (como se citó en Sutz, 1997), proponen usar el término competitividad como la idea que se refiere a la generación y expansión de las capacidades endógenas necesarias para sostener el crecimiento económico y desarrollo nacional dentro de un cuadro de creciente globalización e internacionalización.

Según un estudio realizado por la Dirección general de Política de la PYME, la internacionalización se puede definir como el conjunto de actividades que la empresa desarrolla fuera de los mercados que constituyen su entorno geográfico natural. El grado de internacionalización de una empresa se determina en función de la proporción de actividades que desarrolla en el exterior (Martínez, 2008).

La internacionalización es un proceso complejo, dinámico, planificado. Para ello, importan la definición de objetivos, la capacidad de asumir riesgos, la experiencia de interrelación, el potencial de expansión, la capacidad competitiva. La internacionalización de empresas, amerita tener claro, entre los objetivos de la organización, el interés en la proyección de las actividades. 
Cuadro 1. Teorías y modelos sobre el proceso de internacionalización

$\begin{array}{ll}\text { Modelos tradicionales } & \text { - Teoría de las ventajas monopolísticas } \\ & \text { - Modelo de internalización } \\ & \text { - Enfoque macroeconómico } \\ \text { Modelos de internalización } & \text { - Modelo Uppsala (Johanson y Wiedersheim- } \\ \text { dinámicos o modelos por etapas } & \text { Paul, 1975, Johanson y Vahlne, 1977) } \\ & \text { - El enfoque de innovación } \\ & \text { - Modelo del ciclo de vida del producto de } \\ & \text { - } \text { (1966). } \\ \text { Teorías contemporáneas } & \text { - Enfoque de la estrategia de entrada } \\ & \text { - Enfoque de las redes } \\ & \text { - Empresas Born global }\end{array}$

Elementos estratégicos del proceso de internacionalización

Para internacionalizarse, las empresas deben considerarse como más que un núcleo de producción económica individual. Significa formar parte de una gran economía local, regional, nacional y mundial. Es un proceso que mantiene a las entidades $y$ empresas conectadas con su origen, mientras experimentan nuevos tipos de relaciones y conexiones, sin cambiar ni sustituir las ya existentes. El proceso de internacionalización promueve la excelencia, la especialización y la producción. Cuando este paso es inminente, acompañado de nuevas oportunidades de incursión, sobreviene la expansión. Esta es signo de crecimiento, de amplitud, de presencia con perspectiva global.

A partir de las ideas de Díaz (2016), se resalta en este estudio que en una sociedad marcada por los procesos de globalización, se pueden distinguir los vectores locales y los globales, y enfatiza la necesidad de una cooperación supranacional como instrumento de refuerzo del desarrollo y la estabilidad local.

La glocalización es un término desarrollado en década de 1980 por las prácticas japonesas, en respeto y reconocimiento siempre a su propia cultura, profesada por toda la nación. Según el Diccionario Oxford de Nuevas Palabras (1991) el término "glocal" y el nombre elaborado "glocalización" se han "formado entrejuntando las palabras global y local para hacer una mezcla" (134).

De acuerdo también con ese diccionario, esta idea ha sido "modelada" según el japonés dochakuka (derivada de dochaku, "el que vive en su propia tierra"), y se trata originariamente del principio agrario de adaptar las técnicas de la finca que uno posee a las condiciones locales, pero que también ha sido adoptado en el ámbito japonés de los negocios para la localización global, una visión global adoptada a las condiciones locales. Más específicamente, los términos "glocal" y "glocalización" llegaron a formar parte de la jerga de los negocios durante los años ochenta, si bien el principal lugar de origen fuera de hecho Japón, un país que durante muchísimo 
tiempo ha cultivado rigurosamente el significado espacio-cultural de su propio país, y donde los aspectos generales de su relación entre lo particular y lo universal han recibido históricamente una 6 atención casi obsesiva (Miyoshi et al., 1989, c.p. Robertson, 2000).

Desde el punto comercial, la glocalización engloba el principio de internacionalización, del que ninguna empresa puede escapar como actividad máxima de desenvolvimiento empresarial, sumado al entendimiento de que la globalidad nos da la oportunidad de alcanzar sin límites los rincones del planeta. Reconoce los procesos de apertura de los mercados, ante la liberación de aranceles y la ampliación de los mercados de comercialización y de las formas de distribución sin límites, conllevando a las empresas y a los países a promover procesos de producción para satisfacer un sinnúmero de necesidades, escondidas o disfrazadas por los grandes poderes del comercio mundial.

Parrat (2002) alude al citado proceso de glocalización desde el punto de vista del mercado. Los ámbitos local, nacional e internacional se consideraban a principios de siglo como compartimentados, mientras que el nuevo esquema actual de representación de la empresa y del mundo asocia los tres niveles. Así, toda estrategia de empresa en el mercado mundializado debe ser a la vez global y local, y ello se traduce en lo que los empresarios japoneses expresan a través del neologismo inglés glocalize (Parratt, 2002, p. 7), que hace referencia al concepto de glocalización, entendida como la articulación entre lo global y lo local desde una visión urbana, como una noción que hoy se aplica tanto a la economía (la ciudad como medio económico adecuado para la optimización de sinergias) como a la cultura (las identidades locales y su relación dialéctica con el universalismo informacional de base mediática).
La glocalización supone para él destacar el ámbito urbano y el papel gestor coordinador promotor de los gobiernos locales para la implementación de políticas que tienen en cuenta unos referentes globales y que se posicionan respecto a ellos. En síntesis: globalización más proximidad (Castells c.p. Parratt, 2002).

La glocalización supone para el ámbito urbano, el efecto coordinador-promotor de los gobiernos locales para la implementación de políticas, con fuertes influencias globales, pero con acertadas intenciones de reconstruir lo propio, lo natural de cada región. La glocalización es una propuesta de globalización, más próxima, inmediata, localizada.

Se puede definir, entonces, la glocalización, como la forma de internacionalización que se basa en la localización de la globalidad y la globalización de lo local. El mundo se ve como un todo desde cualquier localidad y a su vez la localidad se hace partícipe de todo lo internacional.

El enfoque de glocalización dirige sus esfuerzos hacia la reconsideración del ámbito local y nacional, con enfoque global. Retoma espacios, y ayuda a ubicar a quienes están fuera del contexto global, de igual manera que a quienes han obviado la presencia local. Se trata de una concepción social, con aire de globalización que, ante todo, reconoce la realidad de la globalización en todos los aspectos del desenvolvimiento mundial haciendo énfasis en rescatar las bases locales que la globalización ha olvidado.

\section{MATERIALES Y MÉTODO}

Este trabajo tuvo como objetivo precisar una propuesta estructural y organizacional de internacionalización de negocios en el contexto de la glocalización, para ser 
aplicada en organizaciones públicas o privadas, empresariales o no empresariales. Se enmarcó dentro del campo de la gerencia, y se desarrolló como un proyecto especial.

En cuanto a las etapas que se siguieron para su desarrollo, en primer lugar se precisaron los aspectos fundamentales de orden conceptual relacionados con el reconocimiento de las formas y los períodos sociales, económicos y políticos, vistos como internacionalización, globalización y glocalización. En segundo lugar, se profundizó en el estudio de los negocios en sus aspectos fundamentales, a partir de una revisión bibliográfica extensa. Por último, un tercer momento implicó el diseño de la propuesta para la glocalización de negocios, Glocal busines.

Las fuentes de información fueron de carácter documental. La propuesta se construyó a partir de la revisión y análisis de autores, con apoyo de la experiencia empresarial de la investigadora, y de las técnicas de diseño y planificación. Para el análisis se utilizaron las técnicas análisis por relaciones y análisis composicional.

El proceso operativo consistió en determinar el esquema general del trabajo, estudiar aspectos esenciales científicos y estructurales, argumentar in extenso sobre los contenidos generales, y diseño de la propuesta.

\section{RESULTADOS Y DISCUSIÓN}

El principal resultado de este trabajo fue el diseño de la propuesta de Glocal business. Glocal business constituye una propuesta de asesoría e intervención de negocios, inscrita en el contexto de la glocalidad, según las condiciones de cada lugar de origen -en relación con cada propuesta de negocios, en particular-, y de acuerdo a las posibilidades y potencialidades del contexto internacional hacia el cual se oriente cada iniciativa.

El objetivo es esta propuesta es promover la orientación, desarrollo y proyección glocal de las organizaciones, mediante acciones de asesoría, consultoría y guiatura, ajustados a cada caso y circunstancia. Entre sus objetivos específicos están:

- Precisar acciones a seguir con respecto a propósitos de glocalización de personas, entidades y organizaciones, públicas y privadas, susceptibles de ser interesadas por el modelo Glocal business.

- Potenciar propósitos gerenciales, administrativos y de proyección formal, de entidades y organismos, tendientes a las acciones de glocalización.

- Ejecutar la propuesta de Glocal business para llevar a cabo la internacionalización de la empresa.

- Efectuar seguimiento y evaluación a los proyectos, iniciativas y entidades inscritos en Glocal business.

Con relación a los destinatarios, Glocal business está dirigida a gerentes de negocios, a asesores, consultores, socios, profesionales, empresarios. A toda persona interesada en ejecutar una satisfactoria experiencia empresarial en contextos internacionales, de variadas condiciones económicas, sociales, culturales y políticas. De igual manera, a todo emprendedor, visionario, entusiasta de los negocios y de las actividades sociales, culturales, económicas, con proyección internacional, sea regional o planetaria. Es una propuesta para personas con corazón amplio, mente abierta, pies sobre la tierra y sueños, aspiraciones y emprendimiento por desarrollar. 
El cuanto a su desarrollo, Glocal business considera cinco pasos esenciales, los cuales deben ser llevados de manera consecutiva, pero que a su vez, son flexibles y permiten una revisión constante entre ellos, lo cual facilita la búsqueda e interpretación de la información adecuándolo a las circunstancias de cada propuesta. Cada uno de estos pasos, identificados seguidamente, contribuye a la búsqueda, análisis, diseño y planteamiento de cada propuesta en particular. Estos pasos son: Descripción, análisis, prospección, ejecución y evaluación.
En el primer paso se describe el escenario de la empresa, entidad, organización, con el fin de determinar los recursos con los que cuenta y las condiciones que la rodean. Se busca reconocer la posición y valor en el mercado en el que se desarrolla, antes de iniciarse en la internacionalización. El objetivo de la descripción es determinar las condiciones específicas y generales que ha tenido y tiene la organización, así como las más inmediatas, de la empresa, institución u organización interesada en Glocal business. Entre los aspectos a describir podemos mencionar:

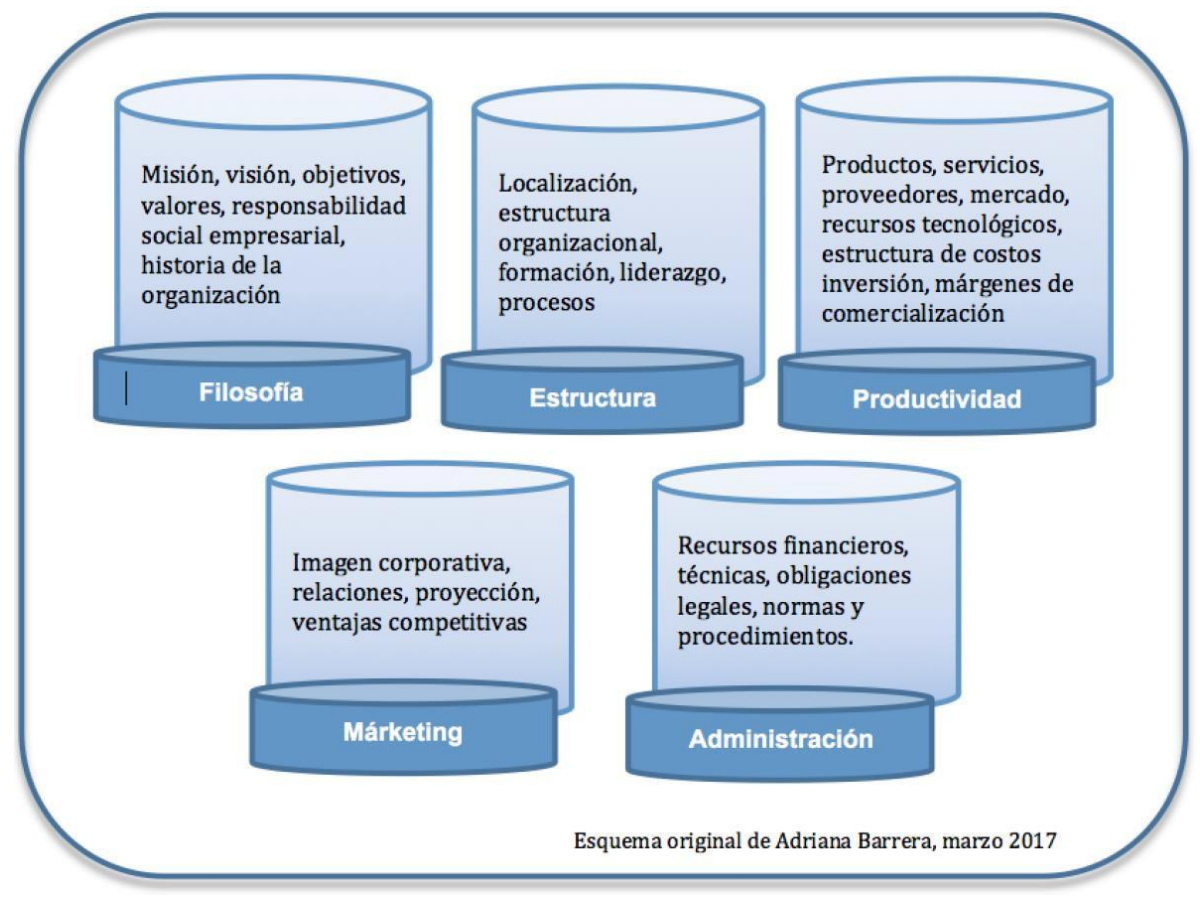

Gráfico 1. Aspectos y fundamentos de la descripción

En la etapa del segundo paso, se analizan las condiciones de índole política, económica y social del escenario glocal donde se pretende llevar a cabo las actividades de la organización. Éstas son importantes para definir sus posibilidades de incursión, crecimiento y desarrollo. En consecuencia, en el análisis se prevén situaciones para afrontar a futuro, se ponderan las variables que favorecen la intención de glocalización y se estiman las consecuencias para la organización. El análisis también permite determinar si la organización se encuentra preparada para enfrentar un proceso de glocalización y de la misma manera permite precisar, tomando en 
cuenta la experiencia de la organización, los aspectos que deben ser reforzados, a fin de preparar la estructura para esta propuesta y definir los tiempos que tomaría llevarlo a cabo. El objetivo del análisis es: Identificar las posibilidades reales de proyección y posicionamiento e inserción en los mercados locales e internacionales, en el contexto de la glocalización.

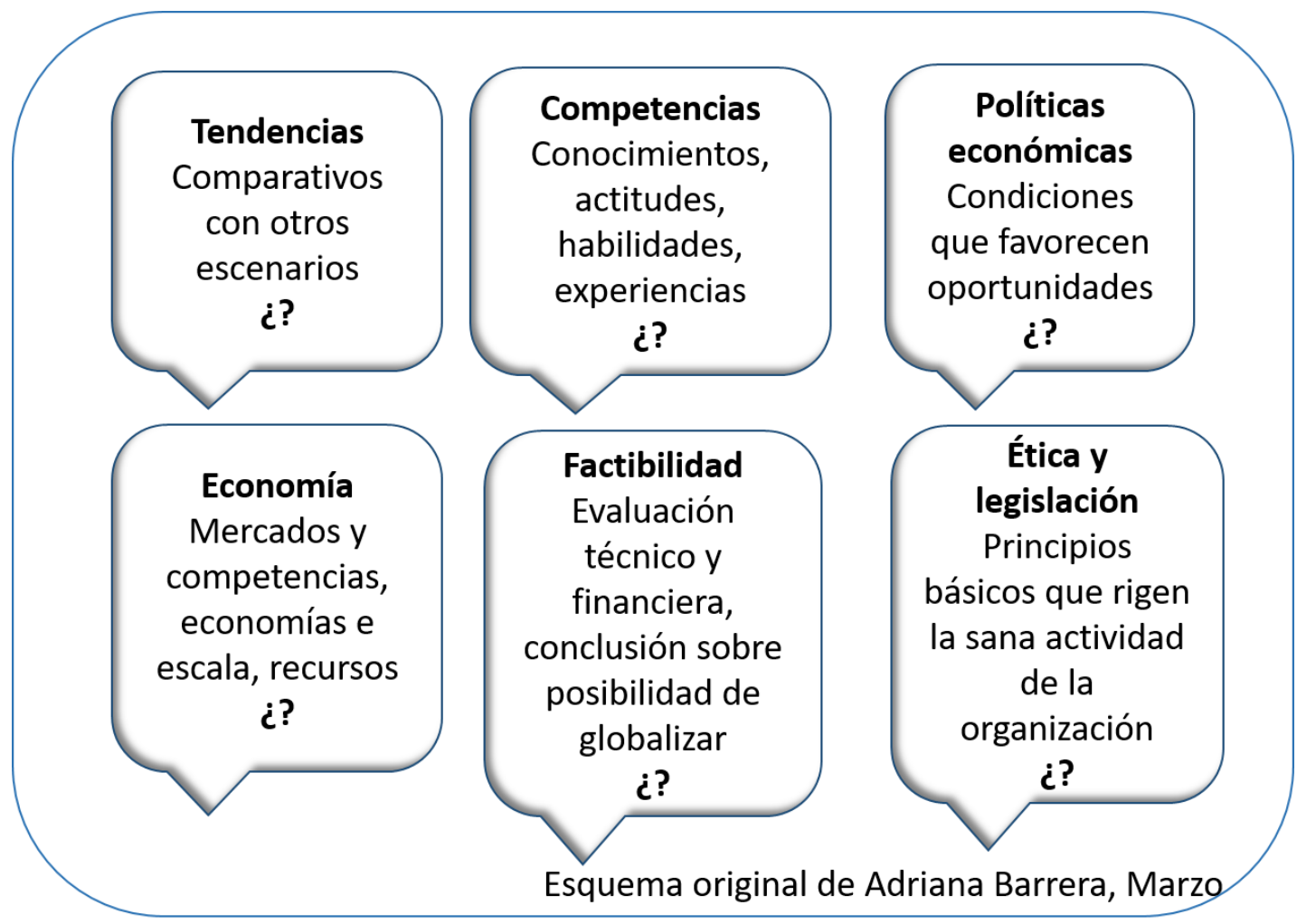

Gráfico 2. Aspectos y fundamentos del análisis

Es en la etapa predictiva, tercer paso, donde se diseña la propuesta estratégica adaptada al nuevo escenario glocal, a fin de preparar las condiciones de la empresa. En este paso se definen las etapas y se determinan los procesos requeridos para la creación, instalación, organización y conformación de la empresa en el nuevo escenario glocal. También se definen los aspectos estratégicos a ser aplicados, tomando en consideración los recursos disponibles en la nueva glocalidad, como la estrategia para enfrentar los posibles escenarios ante proveedores, mercados y clientes. El objetivo de la prospección es elaborar la propuesta técnica y económica de desarrollo, inserción y posicionamiento glocal de la empresa, institución o iniciativa. 
Infograma 1. Aspectos y fundamentos de la prospección

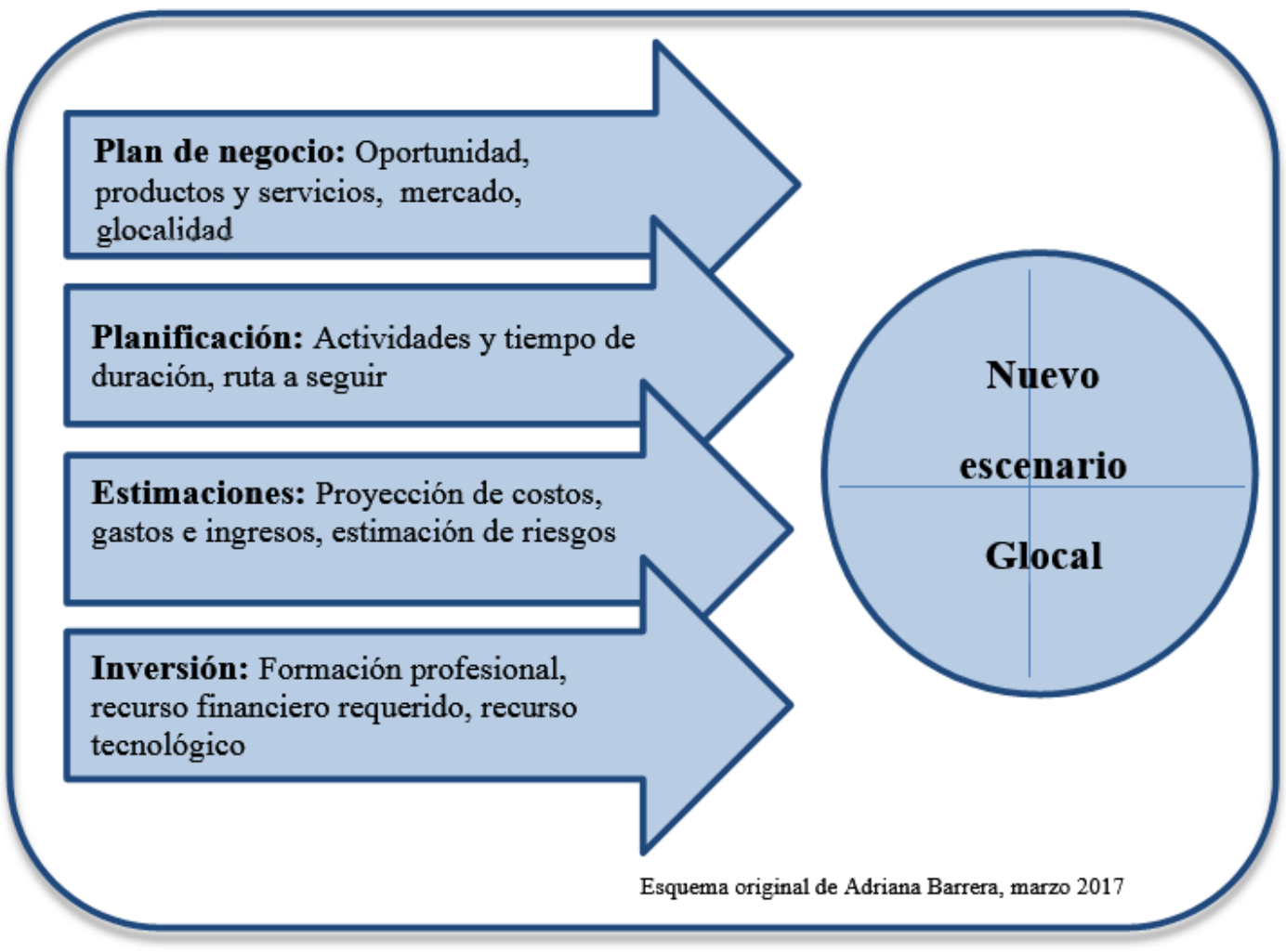

La ejecución es el paso cuarto, paso de la acción, de realización de las iniciativas. Corresponde al desarrollo formal de la propuesta. En él, se procede a ejecutar el plan gerencial de internacionalización en el nuevo escenario glocal, una vez tomada la decisión por los interesados en la propuesta. Las bases de esta ejecución están sustentadas en el buen trabajo prospectivo del nuevo escenario glocal. En la ejecución, se consolidan las bases de la nueva glocalidad y se llevan a cabo las acciones previstas en la planificación. La ejecución conlleva a conformar la estructura legal, operativa y física de la organización, y la promoción en el equipo de trabajo, del éxito esperado por el esfuerzo de todos, para consolidar una idea. En esta ejecución se inician las relaciones con los aliados estratégicos, se dota a la empresa de los recursos necesarios para operar y se conforma la estructura administrativa y organizacional con la cual dará respaldo a las operaciones. El objetivo de la ejecución es poner en práctica la propuesta de Glocal business para llevar a cabo la internacionalización de la empresa en respaldo del cumplimiento de sus fines y propósitos. 


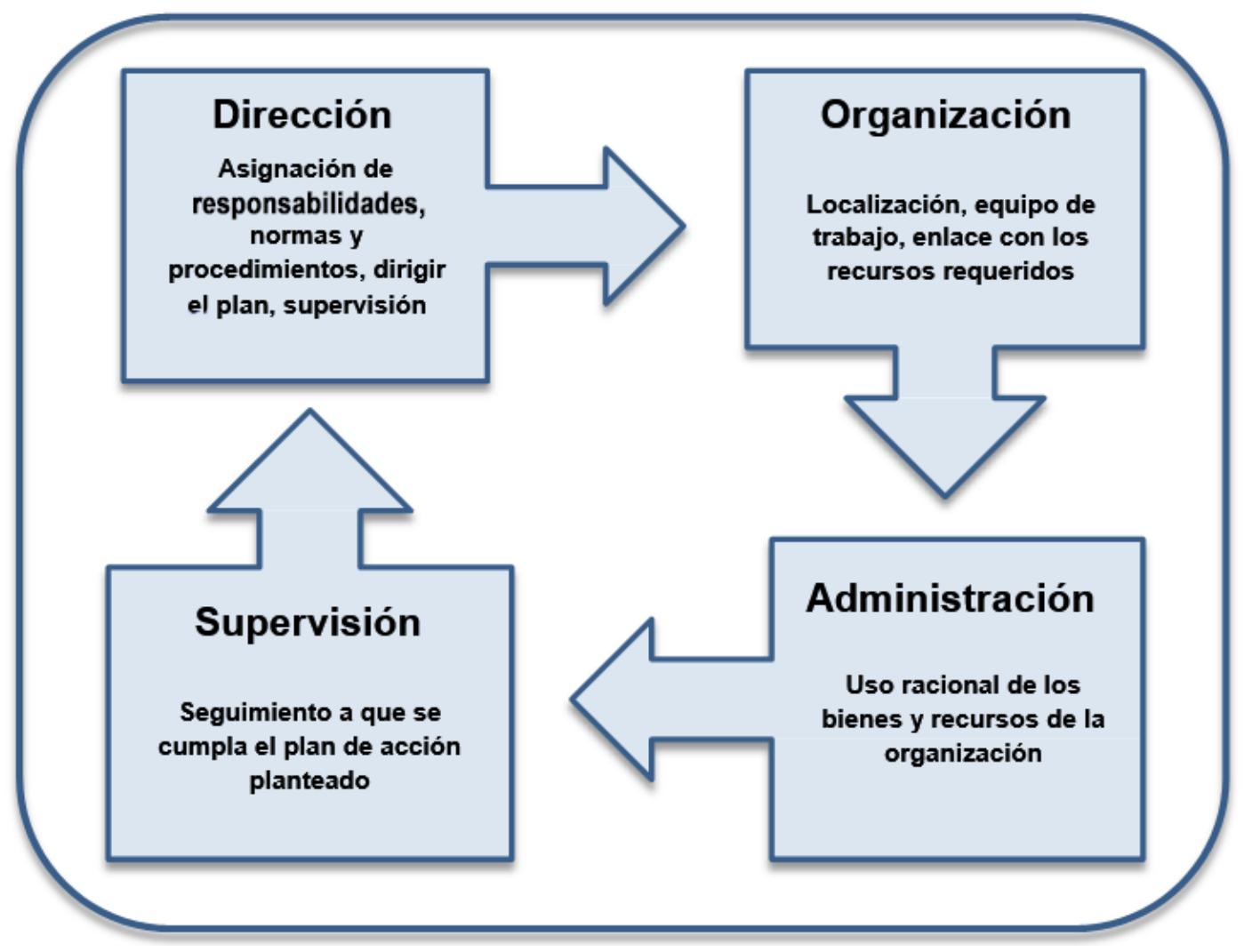

Gráfico 3. Aspectos y fundamentos de la ejecución

Finalmente, corresponde a la evaluación el quinto paso, en sentido global, de igual manera que progresiva, constante; etapa que permite apreciar el estado de la ejecución del proyecto, a lo largo del tiempo estimado como período de estabilización. En ella se hacen las mediciones pertinentes para monitorear los avances de cada una de las actividades requeridas para consolidar la conformación de la empresa en la nueva glocalidad. Parte de la comparación entre lo predictivo y lo ejecutado, a los efectos de evaluar posibles desviaciones $y$ sus consecuencias. El objetivo de esta etapa es evaluar el plan estratégico de internacionalización a lo largo del proceso de ejecución, arranque y estabilización, que facilite tomar decisiones en pro del cumplimiento de los logros. 
Infograma 2. Aspectos y fundamentos de la evaluación

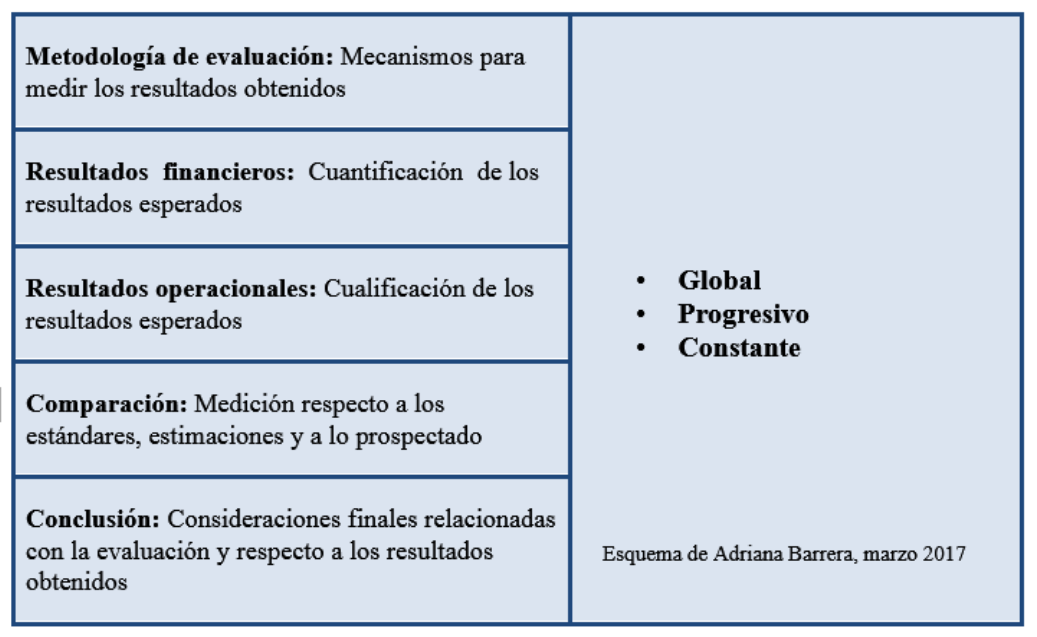

La etapa evaluativa corresponde, entonces, al análisis de la gestión por parte de los ejecutivos y demás personas involucradas en el proyecto, la verificación del cumplimiento de las metas en los tiempos establecidos. Además del análisis periódico de los resultados comparando los gastos, costos e ingresos, versus lo presupuestado. Esta etapa es fundamental ya que permite tomar a tiempo decisiones en caso de que la planificación estimada tenga desviaciones, ya que las mismas pueden tener consecuencias importantes en los resultados finales del proyecto de constitución de la empresa, si no se detectan y se atienden a tiempo.

En general, en la etapa evaluativa se controlan los recursos, los tiempos y los resultados. Es fundamental en ella la comunicación y el análisis oportuno para tomar decisiones coherentes con las circunstancias. El nivel de desviación detectado en esta etapa puede ser consecuencia de un errado análisis predictivo o la selección de recursos y condiciones inapropiados, que, de igual manera, al ser detectado a tiempo evita la pérdida de tiempo y de recursos financieros mayores.

\section{CONCLUSIONES}

A lo largo de este trabajo se logró diseñar la propuesta Glocal business, en sus diferentes componentes. Se articuló justificación, se formularon sus objetivos, se determinaron los destinatarios $y$ se configuraron cada una de las etapas a cubrir para ejecutar la propuesta en cualquier organización.

Glocal business invita incursionar en la práctica del análisis previo y la planificación, el uso de herramientas, claves en las que se requiere confiar para llevar a cabo la ejecución técnica y gerencial del emprendimiento internacional, con ética, responsabilidad y eficiencia. Además, atiende los requerimientos que se presentan en las organizaciones en torno a constructos que ofrecen lineamientos para la elaboración, desarrollo, puesta en marcha de planes, por parte de empresas $u$ organizaciones, con el objetivo de crear las condiciones necesarias para salir al mercado internacional. 
Cuando una se desarrolla, se aprovecha la experiencia, que constituye fuente de conocimiento. Por lo tanto, Glocal business tiene su origen en la búsqueda de escenarios operativos válidos para crear y desarrollar oportunidades empresariales y de negocios, con base en la experiencia empresarial, de la mano de una propuesta directiva.

Uno de los aspectos más importantes de Glocal business es la visión del emprendedor y la intención de llevar sus proyectos, sueños y aspiraciones a un plano internacional, fundamental para que la estructura, y sus aspectos complementarios, vayan cónsonos con las metas. Muchas empresas nacen con visión de internacionalización, pero otras se sumergen en esta intención por razones que las obligan, por cambios de interés en los negocios o por el surgimiento de nuevas oportunidades. Por esto, el enfoque para iniciar este camino es distinto para cada empresa, aspecto este que debe permitir intervenciones con características de originalidad para cada oportunidad, tal y como lo considera Glocal business.

Así mismo, la aplicación de Glocal business aproxima las intenciones de cualquier empresario y emprendedor a la oportunidad de desarrollo, innovación e internacionalización que presenta el mundo de interrelaciones, oportunidades y retos, sin miedo a los fracasos, en la perspectiva de crecimiento, en el marco de los escenarios glocales más diversos y variados. Al ser un propuesta orientada desde los conceptos de globalidad, Glocal business es perfectamente aplicable bajo esquemas de trabajo de alta tecnología, lo cual favorece no sólo su aplicación práctica empresarial y ejecutiva, sino también su divulgación como conocimiento para cualquier contexto cultural y geográfico.

\section{REFERENCIAS}

Barrera, M. (2006). Análisis en Investigación. Editorial Quirón. Caracas, Venezuela

Bustos, A. (2014). Etimología de negocio. Blog de lengua. Extremadura. Disponible: http://blog.lenguae.com/2014/etimologia-de-negocio/ [31.01.2017]

Calva, J. y Álvarez, A. (2007). Globalización y bloques económicos: mitos y realidades. UNAM. México. Disponible: https://books.google.co.ve/books?id=w6 cqjMp5TcEC\&dq=Am\%C3\%ADn, +Samir.+ 1999.+El+capitalismo+en+la+era+de+la+g lobalizaci\%C3\%B3n.+Barcelona:+Paid\%C3 \%B3s\&hl=es\&source=gbs_navlinks_s [05.09.2016]

Díaz, N. (2016). Ulrich Beck (1944). Perfil biográfico y académico. Infoamérica: Revista Iberoaméricana de Comunicación. Málaga. Disponible: http://www.infoamerica.org/teoria/beck 1.htm_[29.04.2016]

Diccionario Oxford de Nuevas Palabras. (1991). Oxford: Oxford University Press

Griffin, R., Ebert, R. (2005). Negocios. Comprensión del sistema de negocios estadounidense. Pearson Educación. México

Hurtado, J. (2010). Guía práctica de metodología de la investigación bajo la comprensión holística de la ciencia. Editorial Quirón. Caracas, Venezuela

Martínez, P. (2008). La internacionalización; el verdadero reto pendiente. Revista de la Facultad de Ciencias Sociales y Jurídicas de Elche, 1(3), 250-264

Naciones Unidas. (2009). Clasificación Industrial Internacional Uniforme de todas las actividades económicas (Ciiu). Informes estadísticos. Serie M, No. 4, Rev 4. Naciones Unidas. Nueva York. Disponible:

http://unstats.un.org/unsd/publication/ seriesM/seriesm_4rev4s.pdf [18.07.2016]

Osterwalder, A. y Pigneur, Y. (2011). Generación de modelos de negocio. Un manual para visionarios, revolucionarios 
y retadores. Grupo Planeta. Barcelona. Disponible:

http://www.generaciondemodelosdenego cio.planetadelibros.com/img/cap01.pdf [17.02.2017]

Parratt, Sonia. (2002). La glocalización de la comunicación. Revista internacional de comunicación. ISSN 1139-1979 No. 7-8

Porter, M. (2016). Índice de progreso social. Social Progress Imperative. Disponible: www.socialprogressimperative.org/data/s pi [25.04.2016]

Robertson, R. (2000). Glocalización: tiempoespacio y homogeneidad heterogeneidad.

Disponible:

https://ivanhistorico.files.wordpress.com/ 2013/12/art_4_glocalizacic3b3n-tiempoespacio.pdf

Sakaya, T. (1995). Historia del futuro: La sociedad del conocimiento. Andrés Bello. Santiago de Chile

Sánchez, R., Contreras, R., y López, L. (2014). Emprendimiento y Mipymes. Nuevo balance y perspectivas. Pearson. México

Sutz, Judith. (1997). Innovación y desarrollo en América Latina. Nueva Sociedad. Caracas

Steimberg, F. (2004). La nueva teoría del comercio internacional y la política comercial estratégica. Eumed.net. Madrid. Disponible:

http://www.eumed.net/cursecon/libreria/ 2004/fs/fs.htm_[23.11.2016] 